\title{
Simulation of Vertical Plane Turbulent Jet in Shallow Water
}

\author{
T. N. Aziz ${ }^{1}$ and A. A. Khan ${ }^{2}$ \\ ${ }^{1}$ Department of Civil Engineering, Clemson University, 110 Lowry Hall, Clemson, SC 29634-0911, USA \\ ${ }^{2}$ Department of Civil Engineering, Clemson University, 218 Lowry Hall, Clemson, SC 29634-0911, USA \\ Correspondence should be addressed to A. A. Khan, abdkhan@clemson.edu
}

Received 11 July 2011; Revised 25 August 2011; Accepted 1 September 2011

Academic Editor: Jianqiao Ye

Copyright ( $) 2011$ T. N. Aziz and A. A. Khan. This is an open access article distributed under the Creative Commons Attribution License, which permits unrestricted use, distribution, and reproduction in any medium, provided the original work is properly cited.

A plane, turbulent, nonbuoyant, vertical jet in shallow water is simulated numerically using a three-dimensional computation model employing standard $k-\varepsilon$ and renormalized group $k-\varepsilon$ turbulent closure schemes. Existing data of mean and turbulent flow quantities, measured using laser Doppler velocimeter, are used to assess the two turbulent closure schemes. Comparisons between the measured and simulated flow field data are made in the free jet region, within the zone of surface impingement, and in the zone of horizontal jets at the surface. The results show that the standard $k-\varepsilon$ scheme performs equally well and in some areas better than the more complicated renormalized group $k-\varepsilon$ scheme in simulating the mean and turbulent flow quantities in this case.

\section{Introduction}

Turbulent jets have been used extensively for discharging effluent into rivers, lakes, and coastal environments. Detailed theoretical and experimental studies of free, turbulent jets (circular or plane jets in an infinite extent of ambient fluid) have been reported by Albertson et al. [1], Abramovich [2], Heskestad [3], and Rajaratnam [4]. The behavior of free, turbulent jets is studied comprehensively using physical models, computational models, and analytical approaches. However, the behavior of jets in a confined environment due to the presence of a free surface and/or solid boundary has been studied only experimentally or numerically.

Gutmark et al. [5] conducted an experimental study to investigate the behavior of a plane turbulent jet impinging on a solid boundary. The boundary was located at a distance of 100-times the nozzle width. The mean and turbulent flow properties were measured using a hot wire anemometer. The structure of vortices was also reported. More recently, Maurel and Solliec [6] experimentally studied impingement of plane turbulent jet on a flat plate located at varying distance from the nozzle. Laser Doppler velocimetry and particle image velocimetry were used to investigate flow properties.

Kuang et al. [7] made a detailed measurement of mean and turbulent flow quantities using laser Doppler velocimeter in a plane jet issuing vertically upward in a still ambient fluid of finite depth. Based on the measured data, four distinct zones were identified and included zone of flow establishment (ZFE), zone of established flow (ZEF), zone of surface impingement (ZSI), and zone of horizontal jet (ZHJ). The configuration and coordinate directions adopted by Kuang et al. [7] along with different zones are shown in Figure 1 . The nozzle width is $d$ and the uniform velocity at the nozzle is given by $w_{0}$. As the shear layer develops the core of the uniform velocity diminishes in width. At a distance of $6 d$ from the nozzle, the shear layer penetrates up to the centerline of the jet [4]. This distance is called the potential core. After the potential core the centerline velocity decays as the jet continues to grow. Outside the potential core, the velocity at the centerline of the jet is denoted by $w_{c}$, and $w$ is the vertical component of the velocity at any point $(x, z)$ in the domain. The horizontal component of the velocity at any point $(x, z)$ is given by $u$.

The free jet regions (ZFE and ZEF) in cases of plane jet impingement on solid surface (Case I) and plane jet issuing vertically upward in a still ambient fluid of finite depth (Case II) are similar. However in ZEF for Case II, Kuang et al. [7] found that the centerline velocities were lower than the free jet and the difference was attributed to the proximity of the water surface. While Beltaos and Rajaratnam [8] showed that centerline velocity for Case I was as given by the free jet. Using the centerline velocity data of Kuang et al. [7] 
and Maurel and Solliec [6] in ZSI for Case II and Case I, respectively, it was found that although the extent of this zone was the same, the centerline velocities were lower in Case I. The stagnation pressure in Case I manifests itself in the form of surface swell in Case II. The stagnation pressure or the surface swell helps turn the flow from vertical to horizontal direction. The maximum horizontal velocity increases within the zone of surface impingement and then decreases as the horizontal jet expands within ZHJ. In addition to the obvious difference in the velocity profile shape in $\mathrm{ZHJ}$ for the two cases (due to the presence of a wall in Case I), the local maximum velocities in $\mathrm{ZHJ}$ were found to be lower for Case I (measured by Wygnanski et al. [9]) than Case II (measured by Kuang et al. [7]). Analysis of the Reynolds stress data from the two cases also showed differences. Thus, the various zones that form in these two cases, although similar, have subtle differences in the flow characteristics and a detail investigation of jet issuing vertically in shallow depth is warranted.

Due to the difficulty of obtaining an analytical solution, especially in the zones of surface impingement and horizontal jets, the problem of a jet issuing vertically upward in shallow water is studied using physical models. Numerical models have been adopted increasingly to study complex flow problems. To numerically model turbulent flows, the selection of an appropriate turbulence scheme is essential. The two most common turbulence closure schemes are the $k-\varepsilon$ and the renormalized group $k-\varepsilon$ (RNG). For free plane turbulent jets the two schemes provide similar results in the zone of established flow with RNG providing better results for the turbulent kinetic energy along the center of the jet [10]. The aim of this study is to assess the applicability of these two turbulence closure schemes in predicting the mean and turbulent flow characteristics of a jet issuing vertically upward in shallow water. The zones of surface impingement (ZSI) and horizontal jets $(\mathrm{ZHJ})$, where both mean and turbulent flow properties are changing rapidly, are of main interest. The computed growth rates, mean flow velocity profiles, and turbulent kinetic energy profiles in different zones of the jet issuing vertically in shallow water are compared with the measured data acquired by Kuang et al. [7].

\section{Mathematical Details}

The mass and momentum equations, which constitute the Reynolds-averaged Navier-Stokes (RANS) equations, are given by

$$
\begin{gathered}
\frac{\partial u_{i}}{\partial x_{i}}=0 \\
\frac{\partial u_{i}}{\partial t}+u_{j} \frac{\partial u_{i}}{\partial x_{j}}=-\frac{1}{\rho} \frac{\partial p}{\partial x_{i}}+\frac{\partial}{\partial x_{j}}\left[\nu \frac{\partial u_{i}}{\partial x_{j}}-\overline{u_{i}^{\prime} u_{j}^{\prime}}\right],
\end{gathered}
$$

where $x_{i}$ represents coordinate directions ( $i=1$ to 3 for $x, y, z$ directions, resp.), $u_{i}$ is the time-averaged velocity component ( $u, v, w$ in $x, y, z$ directions, resp.), $t$ represents time, $\rho$ is the fluid density, $p$ is the piezometric pressure, $v$ is the kinematic viscosity of the fluid, $\overline{u_{i}^{\prime} u_{j}^{\prime}}$ are turbulent normal and shear stresses, and $i$ and $j$ are indices which range from 1 to 3 .

A constitutive relationship is required to relate the turbulent normal and shear stresses to mean flow velocities and is achieved through the use of turbulent eddy viscosity. The relationship is given by

$$
-\overline{u_{i}^{\prime} u_{j}^{\prime}}=v_{t}\left(\frac{\partial u_{i}}{\partial x_{j}}+\frac{\partial u_{j}}{\partial x_{i}}\right)-\frac{2}{3} k \delta_{i j}
$$

where $v_{t}$ is the turbulent eddy viscosity, $\delta_{i j}$ is the Kronecker delta, and $k\left(=0.5 \overline{u_{i}^{\prime} u_{j}^{\prime}}\right)$ is the turbulent kinetic energy per unit mass. The turbulent eddy viscosity is computed using turbulent kinetic energy per unit mass and the dissipation rate of turbulent energy per unit mass $(\varepsilon)$ as follows

$$
v_{t}=c_{\mu} \frac{k^{2}}{\varepsilon}
$$

where $c_{\mu}$ is an empirical coefficient. To determine the turbulent eddy viscosity and hence the turbulent normal and shear stresses, equations for determining the turbulent kinetic energy $(k)$ and dissipation rate $(\varepsilon)$ per unit fluid mass are required.

In the case of the standard $k-\varepsilon$ scheme, the $k$ and $\varepsilon$ are determined from the following two equations

$$
\begin{aligned}
\frac{\partial k}{\partial t}+u_{i} \frac{\partial k}{\partial x_{i}}= & \frac{\partial}{\partial x_{i}}\left(\frac{v_{t}}{\sigma_{k}} \frac{\partial k}{\partial x_{i}}\right)+v_{t}\left(\frac{\partial u_{i}}{\partial x_{j}}+\frac{\partial u_{j}}{\partial x_{i}}\right) \frac{\partial u_{i}}{\partial x_{j}}-\varepsilon \\
\frac{\partial \varepsilon}{\partial t}+u_{i} \frac{\partial \varepsilon}{\partial x_{i}}= & \frac{\partial}{\partial x_{i}}\left(\frac{v_{t}}{\sigma_{\varepsilon}} \frac{\partial \varepsilon}{\partial x_{i}}\right)+c_{1 \varepsilon} \frac{\varepsilon}{k} v_{t}\left(\frac{\partial u_{i}}{\partial x_{j}}+\frac{\partial u_{j}}{\partial x_{i}}\right) \frac{\partial u_{i}}{\partial x_{j}} \\
& -c_{2 \varepsilon} \frac{\varepsilon^{2}}{k}
\end{aligned}
$$

where $\sigma_{k}, \sigma_{\varepsilon}, c_{1 \varepsilon}$, and $c_{2 \varepsilon}$ are empirical coefficients. The standard values of the coefficients $c_{\mu}, c_{1 \varepsilon}, c_{2 \varepsilon}, \sigma_{k}$, and $\sigma_{\varepsilon}$ used in the $k-\varepsilon$ scheme are $0.09,1.44,1.92,1.0$, and 1.3, respectively.

In the $k-\varepsilon$ scheme the eddy viscosity is determined from a single turbulence length scale. This means that the turbulent diffusion is restricted to only that length scale for which eddy viscosity is determined. Eddies in a turbulent flow contain a large range of length scales and the turbulent diffusion occurs at all scales. The energy cascades down from larger to smaller size eddies where it is dissipated by viscosity. At some point in this size scale, the energy dissipation is equal to the energy production and is called the inertial scale. This scale is of an order that can be efficiently handled by current computer technology. The renormalization technique uses the inertial scale to describe all the other scales and produces a model that is statistically equivalent to the original NavierStokes equations. The RNG scheme uses different equations 
for determining the $k$ and $\varepsilon$. These equations, as given by Bischof et al. [11], are

$$
\begin{gathered}
\frac{\partial k}{\partial t}+u_{i} \frac{\partial k}{\partial x_{i}}=\frac{\partial}{\partial x_{i}}\left[\left(\frac{\nu_{\text {eff }}}{P_{r k}}\right) \frac{\partial k}{\partial x_{i}}\right]+v_{t} S^{2}-\varepsilon \\
\frac{\partial \varepsilon}{\partial t}+u_{i} \frac{\partial \varepsilon}{\partial x_{i}}=\frac{\partial}{\partial x_{i}}\left[\left(\frac{\nu_{\text {eff }}}{P_{r \varepsilon}}\right) \frac{\partial \varepsilon}{\partial x_{i}}\right]+c_{1 \varepsilon} \frac{\varepsilon}{k} \nu_{t} S^{2}-c_{2 \varepsilon} \frac{\varepsilon^{2}}{k}-R
\end{gathered}
$$

where $P_{r k}$ and $P_{r \varepsilon}$ are Prandtl numbers for $k$ and $\varepsilon$, respectively, $S$ represents the mean rate of strain, and $\nu_{\text {eff }}$ is given by

$$
v_{\text {eff }}=v_{t}\left(1+\sqrt{\frac{\nu}{v_{t}}}\right)^{2} .
$$

The term $R$ on the right-hand side of (6) constitutes a major difference between the RNG and $k-\varepsilon$ schemes. The $R$ term is a source term that is a function of $S, k$, and $\varepsilon$. This term is given by

$$
R=\frac{c_{\mu} \eta^{3}\left(1-\eta / \eta_{0}\right)}{1+\beta \eta^{3}} \frac{\varepsilon^{2}}{k}
$$

where $\eta_{0}$ and $\beta$ are constants having standard values of 4.38 and 0.012 , respectively, and $\eta=S k / \varepsilon$. The standard values of constants $c_{\mu}, c_{1 \varepsilon}$, and $c_{2 \varepsilon}$ used in the RNG schemes are 0.0845 , 1.42 , and 1.68 , respectively.

The $k-\varepsilon$ and RNG schemes have been used extensively with the RNG model used for flow involving rapid strain (strong local changes) and low Reynolds number flows. The RNG scheme is found to be more sensitive to the rate of strain because of the presence of the source term $R$ [11]. Since renormalized group $k-\varepsilon$ scheme is favored for locally varying flows with rapid strain, a situation that exists within the zone of surface impingent (ZSI) and zone of horizontal jets $(\mathrm{ZHJ})$, it will be of interest to compare the results from the two turbulence schemes within these regions.

\section{Experimental Setup}

In this study, the experimental data collected by Kuang et al. [7] was used to evaluate the accuracy of the two turbulence closure schemes. The layout of a jet issuing vertically in shallow water is shown in Figure 1. The tank used for experimental setup was $6.5 \mathrm{~m}$ long ( $x$-direction), $0.4 \mathrm{~m}$ wide ( $y$-direction), and $0.4 \mathrm{~m}$ high ( $z$-direction). The nozzle was cut across the width of the tank (along $y$-direction). The width of the nozzle $(d)$ was $2 \mathrm{~mm}$, and depth of water above the nozzle $(H)$ was maintained at $26 \mathrm{~cm}$ (with $H / d$ value of 130). The resulting jet was two-dimensional in the $x-z$ plane, that is, the variations of the flow field in the $y$-direction were negligible. Three experiments were conducted at nozzle velocity $\left(w_{\circ}\right)$ of $0.90,1.33$, and $1.75 \mathrm{~m} / \mathrm{s}$ corresponding to the nozzle Reynolds number $\left(w_{\circ} d / \nu\right)$ of $1,560,2,340$, and 3,120, respectively. The measurements of mean and turbulent flow characteristics were made using laser Doppler velocimeter. By choosing appropriate scales for nondimensionalizing, the measurement results were found to be independent of

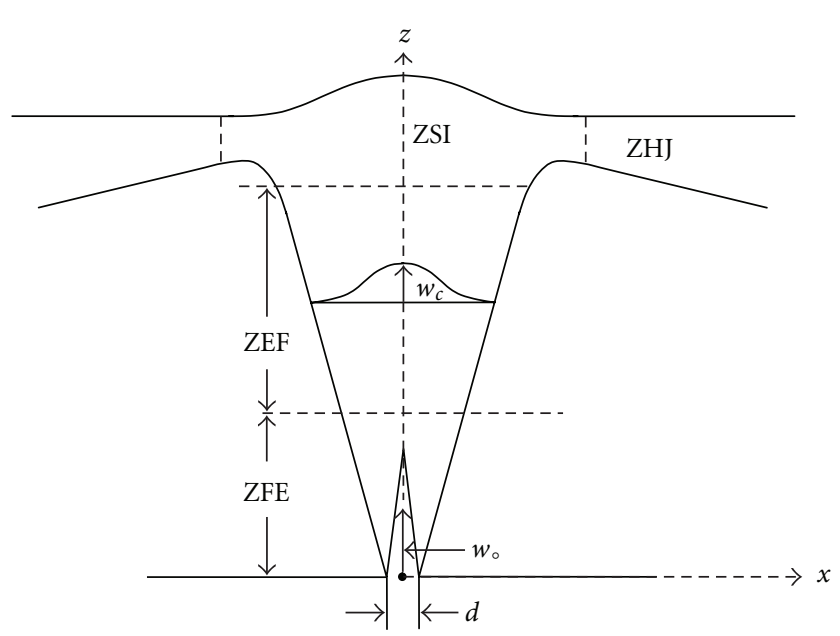

FIGURE 1: Layout of vertical jet in shallow water.

Reynolds number. Complete details regarding experimental setup and results were given by Kuang et al. [7]. The case with the nozzle velocity of $1.33 \mathrm{~m} / \mathrm{s}$ was considered for comparison with the numerical model results and evaluation of the two turbulent schemes.

\section{Numerical Model}

A computational fluid dynamics model, FLOW-3D, developed by Flow Science, Inc., is used to numerically solve the RANS equations. The Flow-3D software provides several options for the turbulent closure scheme including Prandtl mixing length model, two-equation models, and large eddy simulation model. In this study, $k-\varepsilon$ and RNG turbulent closure schemes, with standard coefficients as described earlier, are used. In the Flow-3D model, the Reynolds-averaged Navier-Stokes equations along with the turbulent closure scheme (if needed) are solved using a finite volume/finite difference method in an Eulerian rectangular or cylindrical grid (orthogonal mesh). The model can solve the equations explicitly or implicitly and the momentum advection terms can be approximated using first-order or second-order approximation. In this study, the explicit scheme with second-order accuracy is adopted. The "saw-tooth" representation of the boundaries is eliminated through the use of the fractional area/volume obstacle representation (FAVOR) method [12]. In the FAVOR method, the boundaries are determined independent of the grid generation process. The position of the free surface is predicted using a modified volume of fluid method as part of the solution.

The geometry of the experimental setup, as given by Kuang et al. [7], was adopted for numerical simulation. A boundary condition in terms of velocity of $1.33 \mathrm{~m} / \mathrm{s}$ was specified at the nozzle. The water depth was maintained at $26 \mathrm{~cm}$ above the nozzle through the use of a continuative boundary condition at the tank boundaries in the $x$ direction. The continuative boundary condition kept the water level the same while allowing flow out of the tank by specifying zero velocity gradients at the tank boundaries. 


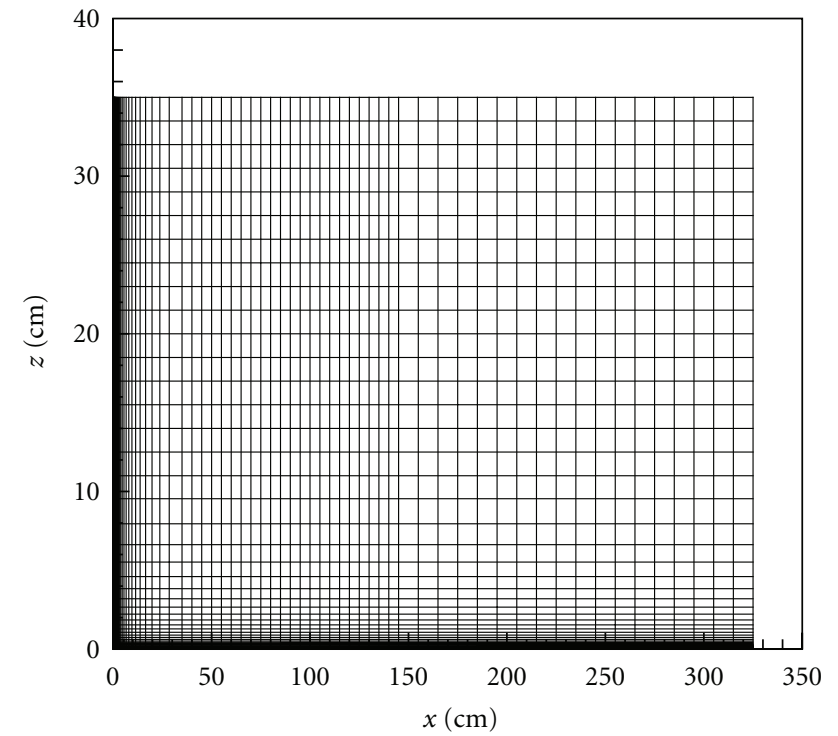

Figure 2: Mesh configuration.

To model the scenario as two-dimensional flow in the FLOW-3D model, only one cell needed to be specified in the $y$-direction. The specification of one cell in the $y$-direction allowed for $2 \mathrm{D}$ computation in the vertical plane. Full 3D computation was also performed using a course mesh and the results were compared with the corresponding $2 \mathrm{D}$ computation. The results from the $2 \mathrm{D}$ and $3 \mathrm{D}$ computations were the same. Since staggered grid was used for computation, where velocities were computed on the cell faces and all other quantities were calculated at the center of the cell, minimum of two cells were needed to determine velocity at the center of the jet. A symmetric mesh was utilized in the $x$-direction on either side of the nozzle. The mesh for the right half of the domain is shown in Figure 2. In the $x$-direction, starting with the cell size of $0.1 \mathrm{~cm}$ within the nozzle, the cell size was increased geometrically by a factor of 1.2 up to a distance of $35 \mathrm{~cm}$ from the center of the nozzle. After which a constant cell size of $5 \mathrm{~cm}$ was used up to a distance of $145 \mathrm{~cm}$. The last segment (between $145 \mathrm{~cm}$ and $325 \mathrm{~cm}$ ) had a cell size of $10 \mathrm{~cm}$. It should be mentioned that the region of interest in terms of comparing computational results with the measured data was within $15 \mathrm{~cm}$ on either side of the nozzle. The computational mesh extent in the vertical direction was set to $35 \mathrm{~cm}$ (the water depth was set to $26 \mathrm{~cm}$ ) to account for the bulge at the water surface. The size of the first cell in the vertical direction was set as $0.1 \mathrm{~cm}$. The cell size was increased geometrically by a factor of 1.2 up to a distance of $11 \mathrm{~cm}$. From then on, a constant cell size of $1.5 \mathrm{~cm}$ was used. The total numbers of cells in the $x$ and $z$-directions were 146 and 43, respectively. Several other mesh sizes were tried to ascertain that the computational results would be mesh independent. It was found that the results obtained using the current mesh and that obtained by doubling the number of cells had difference of less than $1 \%$ (based on the maximum velocity in the ZEF, ZSI, and ZHJ).
TABLe 1: Growth rate and virtual origin comparison.

\begin{tabular}{lccc}
\hline Characteristics & Kuang et al. [7] & $k-\varepsilon$ scheme & RNG scheme \\
\hline Growth rate $(C)$ & 0.1 & 0.104 & 0.117 \\
Virtual origin $\left(z_{\circ} / d\right)$ & 5.0 & 0.51 & -0.61 \\
\hline
\end{tabular}

The computational time step is automatically selected by the model based on stability criterion.

\section{Comparison of Results}

The computational results obtained using the $k-\varepsilon$ and RNG turbulent schemes are compared to the measured data [7] for the nozzle Reynolds number of 2,340. Kuang et al. [7] determined, by analyzing the variation of longitudinal turbulent intensity along the centerline of the vertical jet, that the zone of flow establishment (ZFE) extended up to $z / d$ of 25 and the zone of established flow (ZEF) ranged from $z / d$ of 25 to 100 (analysis would be shown later). The virtual origin of the jet $\left(z_{0} / d\right)$ and the growth rate within the zone of established flow (ZEF) from measured and simulated data are compared in Table 1 . The measured and simulated results show a linear growth of the jet. The growth rates are obtained using the longitudinal velocity $(w)$ profiles across the jet at various locations and determining the position, $b$, from the centerline where the velocity is half the centerline velocity. By fitting a straight line of the form $b / d=C\left(z-z_{\circ}\right) / d$ to the data, the growth rate, $C$, and the virtual origin, $z_{\circ}$, are determined. The growth rate of the jet using the $k-\varepsilon$ scheme compares well with the measured data while the RNG scheme shows a higher growth rate for the jet. The $k-\varepsilon$ scheme provides virtual origin that is in front of the jet, similar to the measured data. The virtual origin of the jet simulated using the RNG scheme is behind the nozzle. The higher growth rate and the location of the virtual origin in the case of the RNG scheme are the result of wider shear layer predicted by the RNG scheme. This is the direct result of a higher sensitivity of the eddy viscosity to the strain rate.

The variation of longitudinal velocity $\left(w_{c}\right)$ and turbulent kinetic energy per unit mass $(k)$ along the centerline of the jet are shown in Figures 3 and 4, respectively. Although both schemes estimate higher growth rate for the jet in ZEF, the longitudinal velocity along the centerline of the jet is overpredicted by the two schemes when compared to the measured data. The centerline velocity predicted by the RNG scheme is more accurate than that predicted by the $k-\varepsilon$ scheme. The turbulent velocity fluctuations along the center line of the jet, as shown in Figure 4, show that both schemes predict the ZEF within the range of $20 \leq$ $\left(z-z_{\circ}\right) / d \leq 100$. However, within this zone, the turbulent velocity fluctuations are overpredicted by both schemes (the $k-\varepsilon$ scheme providing relatively better accuracy). As shown by the measured data and the two schemes, the turbulent velocity fluctuations increase rapidly beyond $\left(z-z_{\circ}\right) / d$ of 100 (the zone of surface impingement).

The variations of longitudinal $(w)$ velocity profiles across the width of the jet at different locations along the jet are shown in Figures 5-7 (the data for the RNG scheme is 


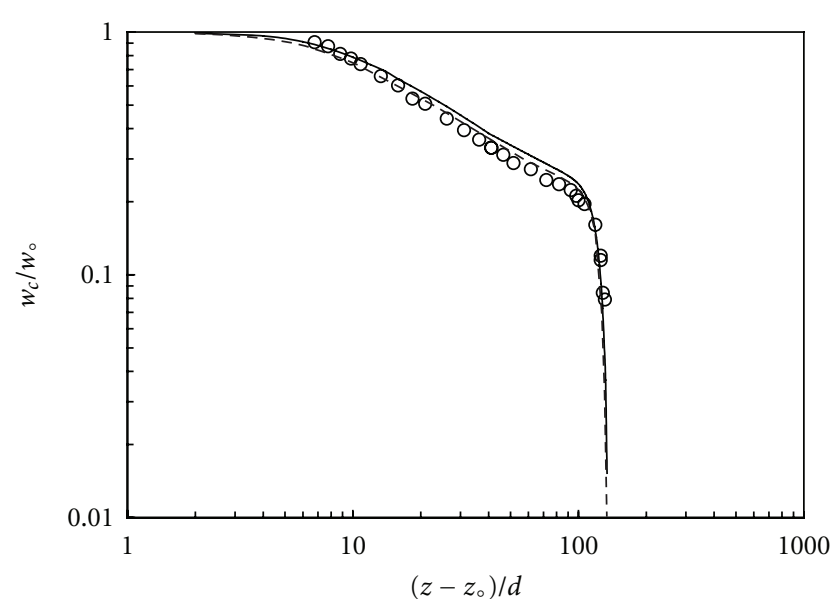

$\begin{array}{ll}- & k-\varepsilon \text { scheme } \\ -- & \text { RNG scheme } \\ \circ & \text { Kuang et al. }\end{array}$

FIgURE 3: Centerline velocity variation.

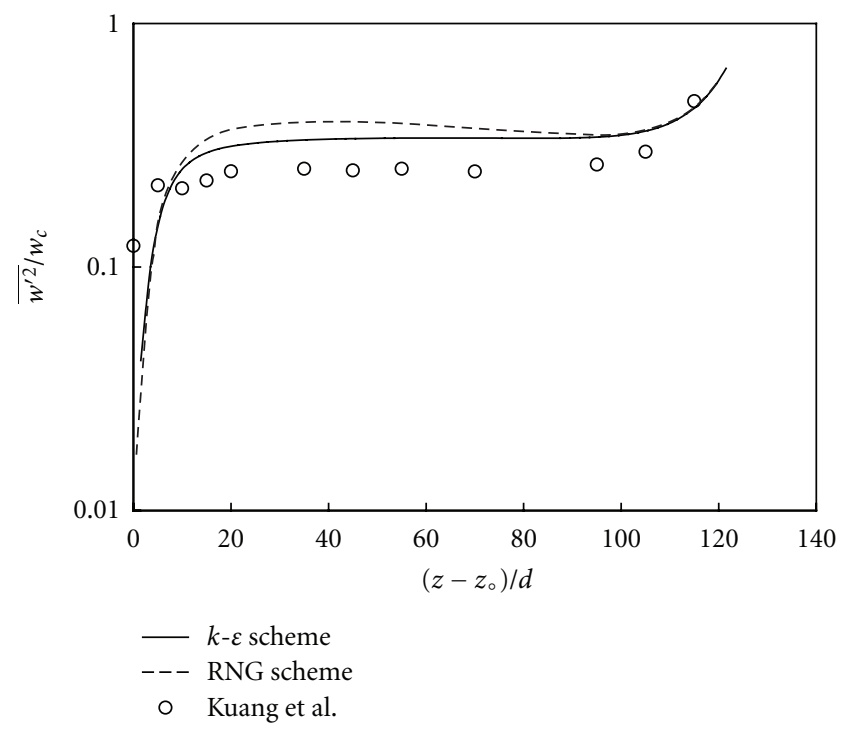

FIGURE 4: Variation of centerline turbulent velocity fluctuations.

offset by 0.5 ). In each case, the predicted velocity profiles are compared with the theoretical curve and/or with a typical measured velocity profile within that zone. The simulated longitudinal velocity profiles in the ZFE are compared to the measured data and a theoretical curve for ZEF in Figure 5. The theoretical curve that fits the measured data [7] in the zone of established flow is given by

$$
\frac{w}{w_{c}}=\operatorname{sech}^{2}\left(\frac{x}{0.134\left(z-z_{\circ}\right)}\right),
$$

where $z_{\circ}=5 d$, as determined by the measured data, is used. The simulated velocity profiles using the $k-\varepsilon$ scheme agree well with a typical measured profile in the ZFE and are below the theoretical line. The velocities are overpredicted by the RNG scheme within the ZFE when compared to the typical

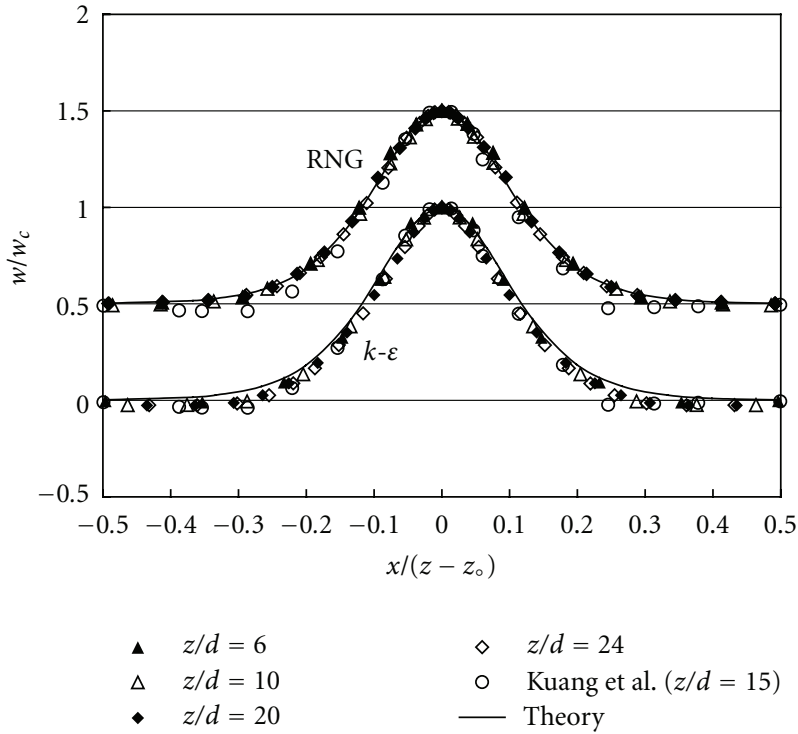

FIGURE 5: Profiles of longitudinal velocity in ZFE.

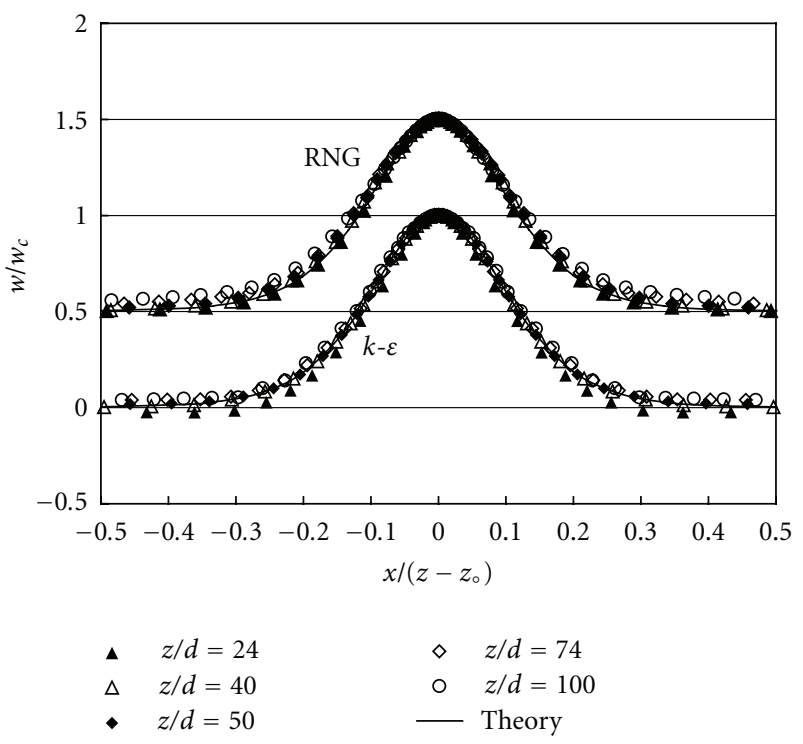

FIgURE 6: Profiles of longitudinal velocity in ZEF.

measured velocity profile and approach the theoretical curve with the increasing longitudinal distance from the nozzle. The simulated velocity profiles within the ZEF, using the $k-\varepsilon$ and RNG schemes, are shown in Figure 6. The measured velocity profiles in this region, as shown by Kuang et al. [7], agree well with (9). This equation is shown in the figure for comparison with the simulated data. The velocity profiles, predicted using the $k-\varepsilon$ scheme, compare well with the theoretical curve while the velocities are overpredicted by the RNG scheme (the error increasing with increasing distance). The higher shear rate and higher turbulence energy predicted by the RNG scheme result in an overprediction of the vertical velocities and growth rate of the jet in the ZFE and ZEF. In the 


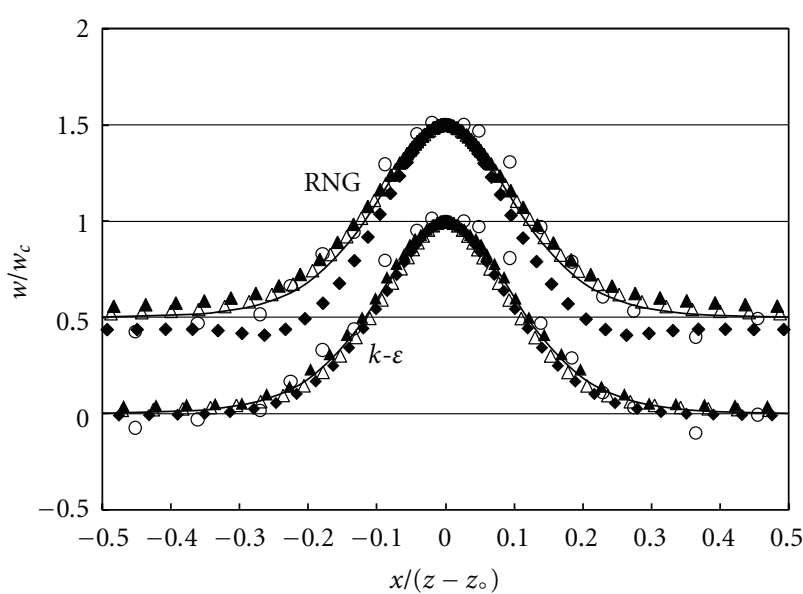

$\triangle \quad z / d=100$

$\triangle \quad z / d=110$

- $z / d=120$

FIgUre 7: Profiles of longitudinal velocity in ZSI.

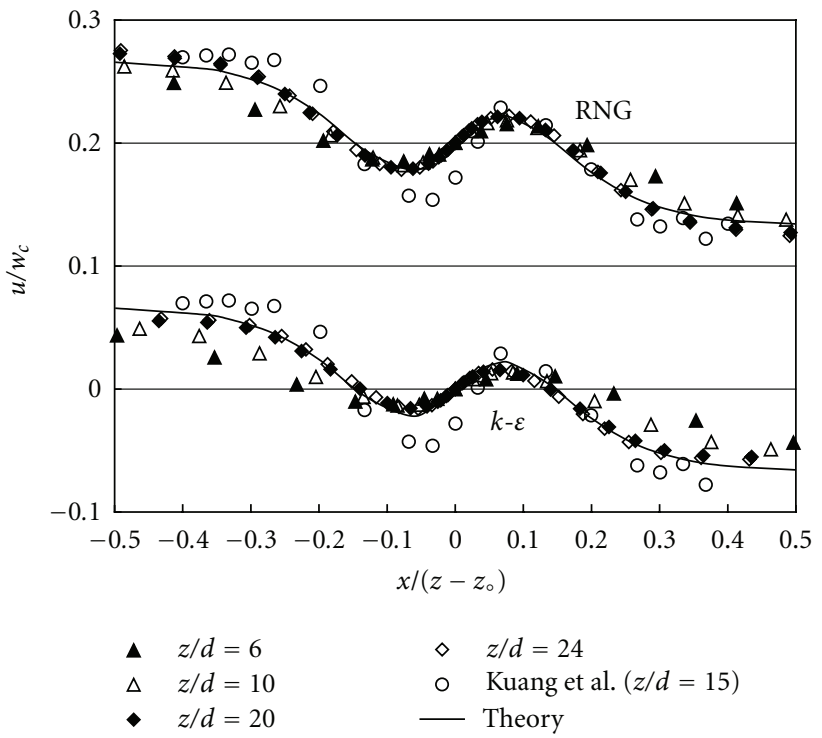

Figure 8: Profiles of lateral velocity in ZFE.

ZSI, as shown in Figure 7, velocities are underpredicted by both schemes. However, in the case of the RNG scheme, the shape of the velocity profile and the magnitude of velocities near the surface $(z / d=120)$ are not accurately predicted.

The simulated results of the lateral velocity profiles across the width of the jet are compared to the measured and/or theoretical velocity profiles in Figures 8-10 (the RNG results in ZEF and ZFE are offset by 0.2 , and by 2.0 in ZSI). Since the velocity profiles are symmetrical about the center of the jet, the following description considers only the left side of the jet. In the ZFE (Figure 8), both schemes underpredict the velocity; however, the accuracy gets better with the increase in the longitudinal distance from the nozzle. The lateral

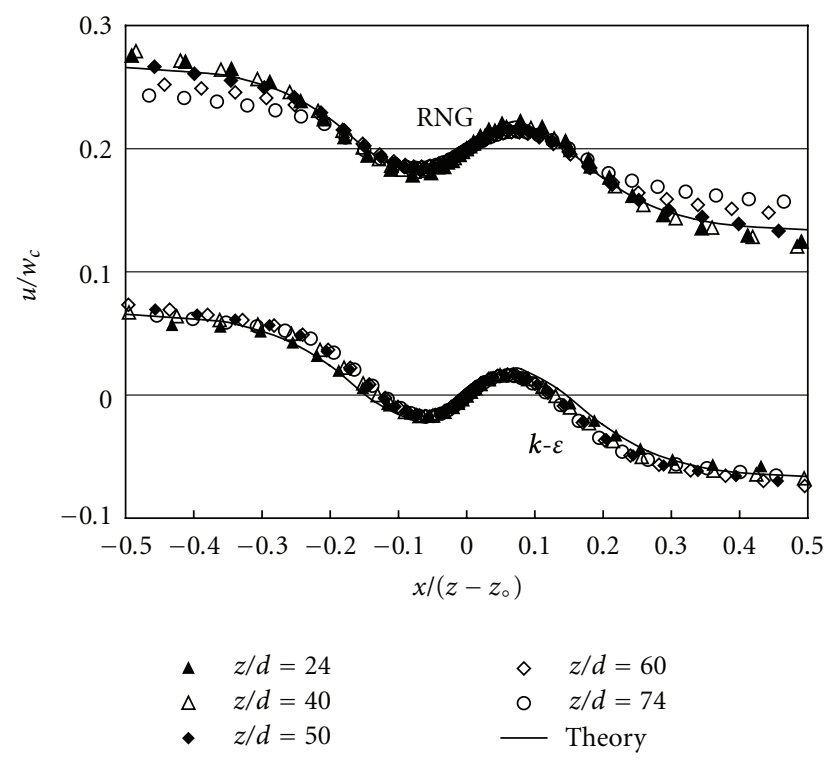

FIgURE 9: Profiles of lateral velocity in ZEF.

velocity at the center of the jet should be zero based on the symmetry of the jet. However, the measured data show some discrepancy near the center of the jet. In the figures, the theoretical curve is given by [7]

$$
\begin{aligned}
\frac{u}{w_{c}}= & \frac{x}{\left(z-z_{\circ}\right)} \operatorname{sech}^{2}\left(\frac{x}{0.134\left(z-z_{\circ}\right)}\right) \\
& -0.067 \tanh \left(\frac{x}{0.134\left(z-z_{\circ}\right)}\right),
\end{aligned}
$$

where $z_{\circ}=5 d$ is used. In the ZEF zone (Figure 9), the lateral velocity profiles predicted by the $k-\varepsilon$ scheme agree well with (10), while in the case of the RNG scheme, velocity profiles are progressively underpredicted with increasing longitudinal distance.

The zone of surface impingement is where the flow turning and acceleration take place. Starting with zero horizontal velocity, the $u$ velocity increases within this zone with horizontal distance. At the same time the vertical velocity diminishes within this zone. This area is associated with high strain rate and, as shown in Figure 10, the horizontal velocity profiles within this area are predicted more accurately by the RNG scheme, especially for $z / d$ of 110 and 120 .

The predicted turbulent kinetic energy (TKE) profiles across the jet at different locations along the jet are shown in Figures 11-13 (the RNG results in the ZFE and ZEF are offset by 0.05 ). In the ZFE (Figure 11), the $k-\varepsilon$ scheme accurately predicts the shape and magnitude of the TKE; however, the magnitude of the TKE is overpredicted outside the zone of $-0.1 \leq x /\left(z-z_{\circ}\right) \leq 0.1$, especially close to the nozzle $(z / d=10)$. The RNG scheme overpredicts by $30 \%$ the TKE near the center of the jet for $z / d=24$. In the ZEF, as shown in Figure 12, the TKE profiles predicted by the $k-\varepsilon$ scheme are self-similar, a property shown by the measured data of Kuang et al. [7] as well. The TKE predicted by 


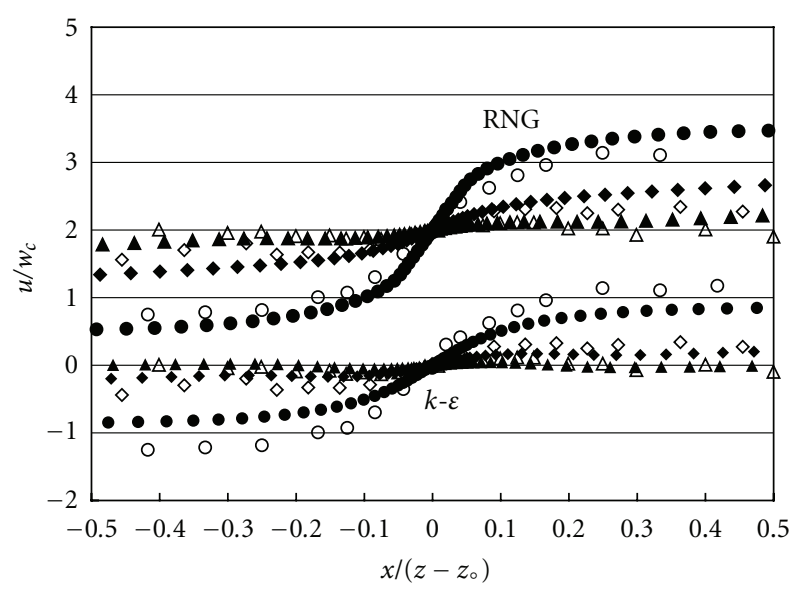

\ $z / d=100$

- $z / d=110$

- $z / d=120$

$\Delta$ Kuang et al. $(z / d=100)$

$\diamond$ Kuang et al. $(z / d=110)$

$\circ$ Kuang et al. $(z / d=120)$

FIgure 10: Profiles of lateral velocity in ZSI.

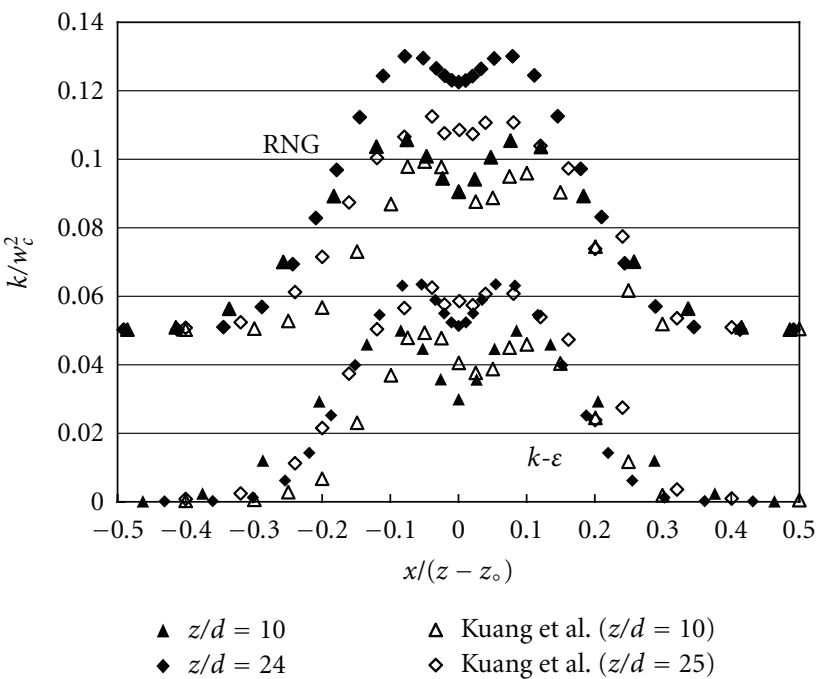

FIGURE 11: Profiles of turbulent kinetic energy in ZFE.

the $k-\varepsilon$ scheme compares well with the measured data in the center part of the jet $\left(-0.1 \leq x /\left(z-z_{\circ}\right) \leq 0.1\right)$, and the TKE is underpredicted outside of this zone. The TKE profiles predicted by the RNG scheme do not show similarity. The TKE is overpredicted near the center of the jet for low values of $z / d$. This may be the result of higher sensitivity of the TKE production on the velocity gradients in the case of the RNG scheme. In the zone of surface impingement, as shown in Figure 13 for $z / d=110$, the TKE predicted by both schemes agrees well with the measured data near the center of the jet; however, the values are underpredicted in the outer part of the jet.

The variations of lateral velocity in the zone of horizontal jets $(\mathrm{ZHJ})$ predicted by the $k-\varepsilon$ and RNG schemes are

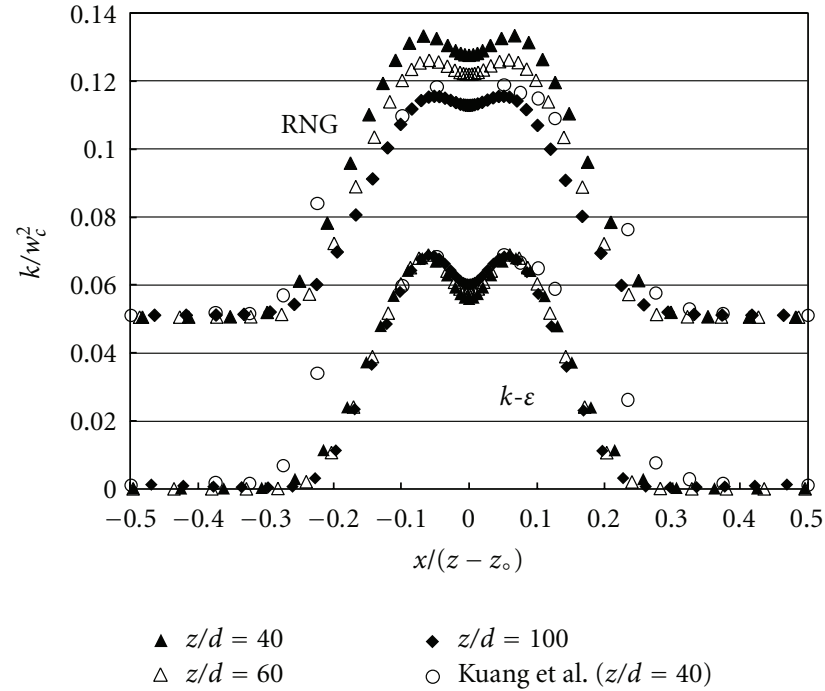

Figure 12: Profiles of turbulent kinetic energy in ZEF.

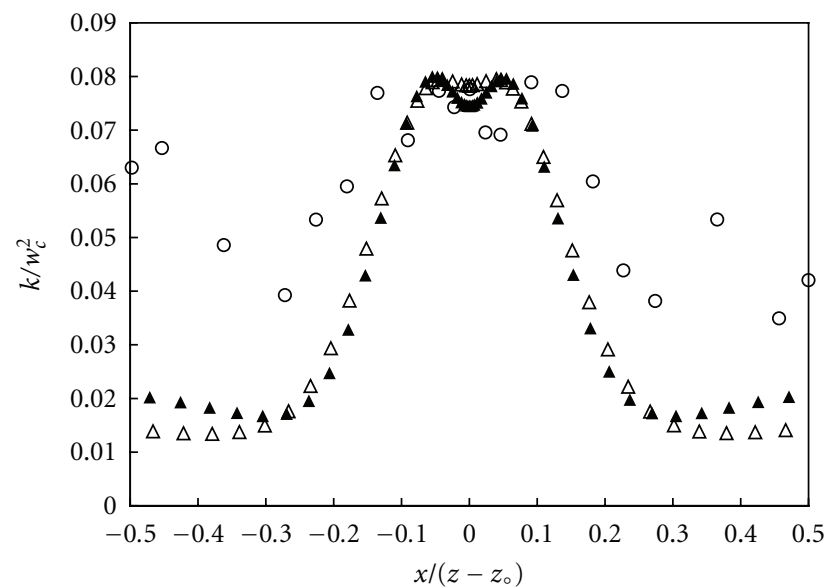
- $k$ - $\varepsilon$ scheme
$\triangle$ RNG scheme
○ Kuang et al.

FIgURe 13: Profiles of turbulent kinetic energy in ZSI $(z / d=110)$.

shown in Figures 14 and 15, respectively. Both schemes predict maximum velocity at the surface and similarity of the velocity profiles in the positive flow region. The results obtained using the $k-\varepsilon$ scheme show a better agreement with the measured data and a Gaussian fit. Kuang at al. [7] provided a Gaussian fit for approximating the measured data in the positive flow region and is given by

$$
\frac{u}{u_{m}}=\exp \left[-6.73\left(1-\frac{z}{H}\right)^{2}\right]
$$

where $u_{m}$ is the maximum lateral velocity. The recirculation zone underneath the surface jets is poorly resolved by the RNG scheme and may be the results of high growth rate 


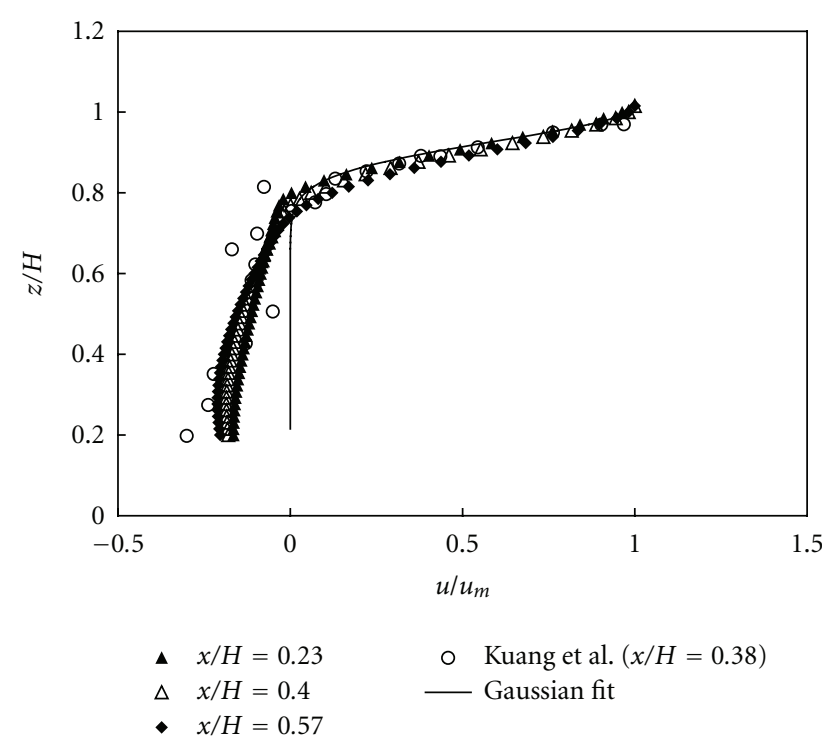

FIgure 14: Profiles of lateral velocity in ZHJ ( $k-\varepsilon$ scheme).

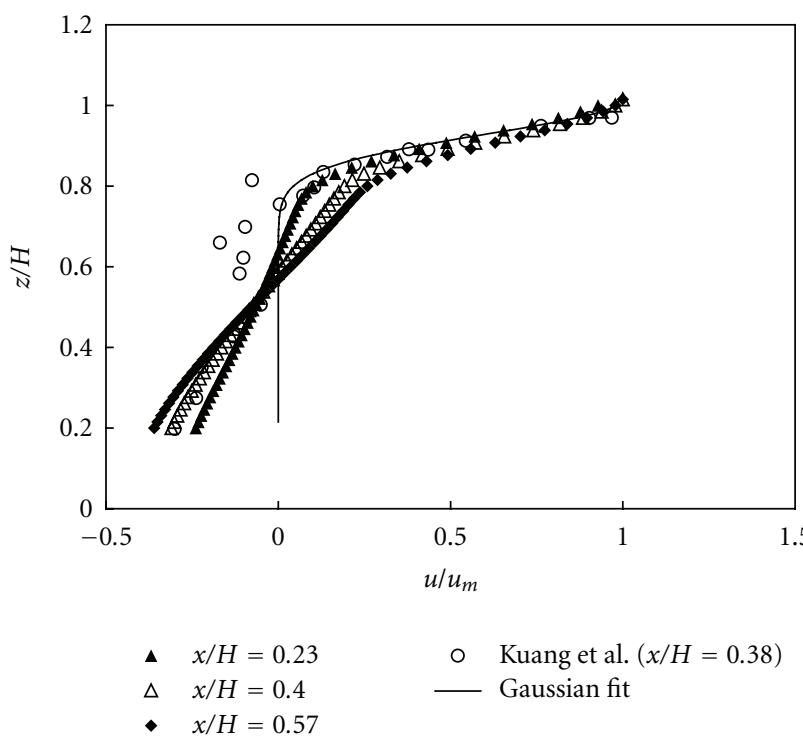

Figure 15: Profiles of lateral velocity in ZHJ (RNG scheme).

of the jet in the ZEF predicted by the scheme. The large width of the jet will interfere with the recirculation flow beneath the surface jets. Kuang et al. [7] reported that the recirculation zone covered the whole depth and its width was about $2 \mathrm{H}$ to $2.5 \mathrm{H}$. The recirculation zone predicted using the two schemes extended the full depth of the tank and had a length of approximately $2.2 \mathrm{H}$. The recirculation zone predicted using the RNG scheme, is shown in Figure 16. A bulge in the water surface, due to the interaction of the jet with the free surface, is clearly visible in the figure. The variations of the maximum lateral velocity $\left(u_{m}\right)$ for both schemes are shown in Figure 17 along with the fit used by

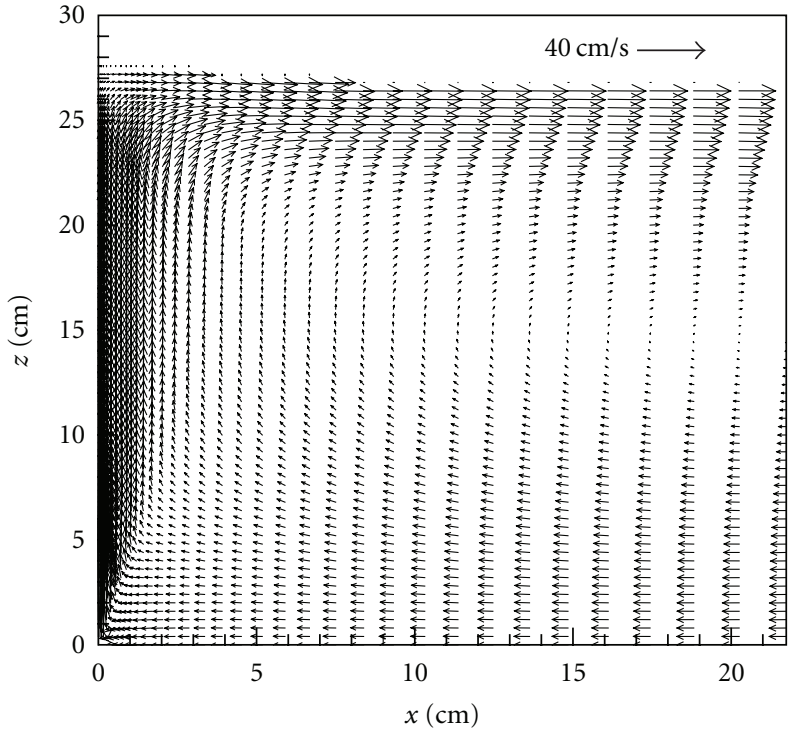

FIGURE 16: Recirculation zone predicted using RNG scheme.

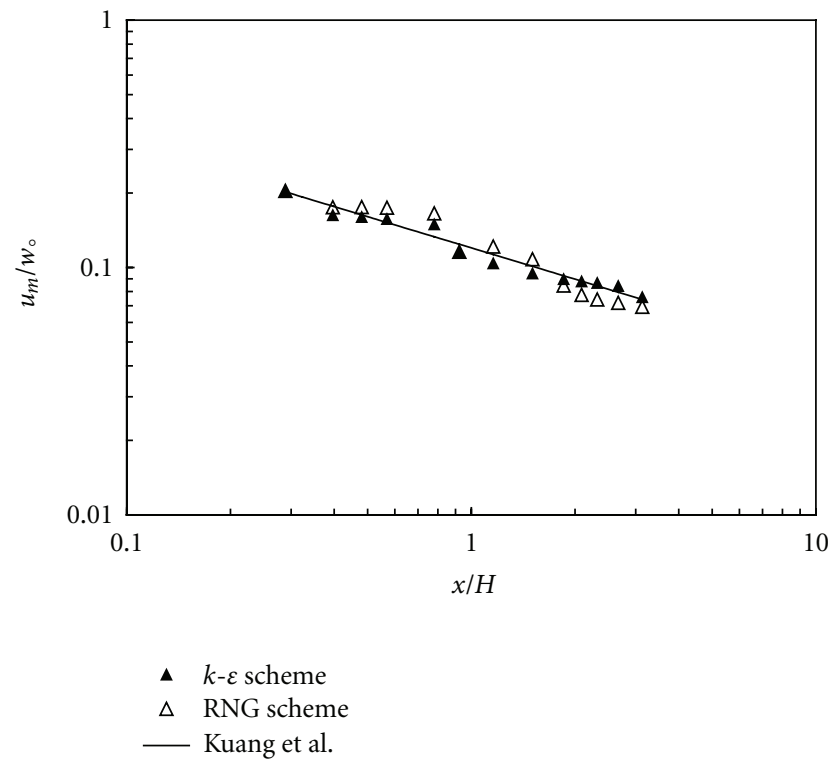

Figure 17: Decay of maximum lateral velocity in ZHJ.

Kuang et al. [7] to describe the measured data. The fit is given by

$$
\frac{u_{m}}{w_{\circ}}=0.11\left(\frac{x}{H}\right)^{-0.47}
$$

The predicted profiles of maximum lateral velocity show oscillations across the linear trend line and are similar to oscillations observed in the measured data.

\section{Conclusions}

A plane turbulent jet issuing vertically upward in shallow water depth has been simulated using a three-dimensional 
computational model employing the $k-\varepsilon$ and RNG turbulent closure schemes. To evaluate the performance of these two schemes, the simulated results from these two schemes using standard coefficients are compared with the data measured by Kuang et al. [7]. The centerline vertical velocities are better predicted by the RNG scheme. The turbulent kinetic energy in ZFE, ZEF, and ZSI is overpredicted by both schemes, with $k-\varepsilon$ scheme providing better agreement with the measured data. The growth rate in the ZEF predicted using the RNG scheme is higher and the location of the virtual origin is behind the nozzle. This is the result of a wider shear layer predicted by the RNG scheme. The growth rate is predicted accurately by the $k-\varepsilon$ scheme and the location of the virtual origin is in front of the nozzle as indicted by measured data.

In the ZFE and ZEF, the vertical velocities are overpredicted by the RNG scheme. The lateral velocities are underpredicted by both schemes in the ZFE with RNG scheme providing a better fit. While in the ZEF, the RNG scheme progressively underpredicts the lateral velocity with an increase in distance from the nozzle. The TKE is overpredicted (by about 30\%) near the center of the jet by the RNG scheme in ZFE. The TKE profiles predicted using the RNG scheme do not preserve self-similarity shown by the measured data and the values are overpredicted near the center. The $k-\varepsilon$ scheme overpredicts TKE at the outer edges of the jet in ZFE. It preserves the similarity of TKE profiles in ZEF; however, the values are underpredicted at the edges of the jet.

The onset of ZSI, based on the centerline variation of TKE, is accurately predicted by both schemes. The vertical velocities are underpredicted by both schemes in this zone, with RNG unable to accurately predict the shape of the profile near the surface. The lateral velocities are predicted more accurately by the RNG scheme in ZSI. The TKE is predicted accurately near the center of the jet and underpredicted at the outer edges by both schemes in this zone.

In the zone of horizontal jets, the lateral velocity in the forward direction and the recirculation zone underneath the surface jet are better predicted by the $k-\varepsilon$ scheme. The larger jet size predicted by the RNG scheme in ZEF may be the reason for poor agreement of the velocity data in the recirculation zone. The decay of the maximum velocity is predicted accurately by both schemes. The $k-\varepsilon$ scheme, albeit simple, provides more accurate results of the flow field for this problem.

\section{Symbols}

$b$ : A measure of the jet growth

C: Growth rate of the jet

$c_{1 \varepsilon}$ : Empirical coefficient

$c_{2 \varepsilon}:$ Empirical coefficient

$c_{\mu}$ : Empirical coefficient

$d$ : Width of the nozzle

$H$ : Water depth in the tank $(26 \mathrm{~cm})$

$k$ : Turbulent kinetic energy per unit mass

$p$ : Piezometric pressure

$P_{r k}$ : Prandtl number for $k$
$P_{r \varepsilon}:$ Prandtl number for $\varepsilon$

$R: \quad$ Source term in RNG scheme

$S: \quad$ Mean rate of strain

$t: \quad$ Time

$u_{i}$ : Time-averaged velocity components

$(i=1,2,3)$

$u$ : Velocity in the $x$-direction at any point in the jet

$u_{m}$ : Maximum lateral velocity in the zone of horizontal jets

$\overline{u_{i}^{\prime} u_{j}^{\prime}}$ : Turbulent normal and shear stresses

$w$ : Velocity in the $z$-direction at any point

$w_{c}$ : Centerline velocity in the $z$-direction

$w_{\circ}$ : Jet velocity at the nozzle

$x$ : Coordinate direction

$x_{i}$ : Coordinate directions in $x, y, z(i=1,2,3)$

$z: \quad$ Coordinate direction

$z_{\circ}$ : Distance to the virtual origin

$\beta$ : A constant

$v \quad$ Kinematic viscosity of the fluid

$v_{\text {eff: }}$ Combination of the fluid and turbulent kinematic viscosity

$v_{t}: \quad$ Turbulent eddy viscosity

$\rho: \quad$ Density of fluid

$\delta: \quad$ Kronecker delta

$\varepsilon: \quad$ Dissipation rate of turbulence per unit mass

$\sigma_{k}: \quad$ Empirical coefficient

$\sigma_{\varepsilon}: \quad$ Empirical coefficient

$\eta$ : $\quad$ A function of $S, k$, and $\varepsilon$

$\eta_{\circ}:$ A constant.

\section{References}

[1] M. L. Albertson, Y. B. Dai, R. A. Jensen, and H. Rouse, "Diffusion of submerged jets," Transaction American Society of Civil Engineers, vol. 115, pp. 639-697, 1950.

[2] G. N. Abramovich, The Theory of Turbulent Jets, MIT Press, Cambridge, Mass, USA, 1963.

[3] G. Heskestad, "Hot-wire measurements in a plane turbulent jet," Journal of Applied Mechanics, vol. 32, pp. 721-734, 1965.

[4] N. Rajaratnam, Turbulent Jets, Elsevier, New York, NY, USA, 1976.

[5] E. Gutmark, M. Wolfshtein, and I. Wygnanski, "The plane turbulent impinging jet," Journal of Fluid Mechanics, vol. 88, no. 4, pp. 737-756, 1978.

[6] S. Maurel and C. Solliec, "A turbulent plane jet impinging nearby and far from a flat plate," Experiments in Fluids, vol. 31, no. 6, pp. 687-696, 2001.

[7] J. Kuang, C. T. Hsu, and H. Qiu, "Experiments on vertical turbulent plane jets in water of finite depth," Journal of Engineering Mechanics, vol. 127, no. 1, pp. 18-26, 2001.

[8] S. Beltaos and N. Rajaratnam, "Plane turbulent impinging jets," Journal of Hydraulic Research, vol. 11, no. 1, pp. 29-59, 1973.

[9] I. Wygnanski, Y. Katz, and E. Horev, "On the applicability of various scaling laws to the turbulent wall jet," The Journal of Fluid Mechanics, vol. 234, pp. 669-690, 1992. 
[10] T. N. Aziz, J. P. Raiford, and A. A. Khan, "Numerical simulation of turbulent jets," Engineering Applications of Computaitonal Fluid Mechanics, vol. 2, no. 2, pp. 234-243, 2008.

[11] C. H. Bischof, H. M. Bucker, and A. Rasch, "Sensitivity analysis of turbulence models using automatic differentiation," Journal on Scientific Computing, vol. 26, no. 2, pp. 510-522, 2005.

[12] J. F. Rodriguez, F. A. Bombardelli, M. H. Garcia, K. M. Frothingham, B. L. Thoads, and J. D. Abad, "High-resolution numerical simulation of flow through a highly sinuous river reach," Water Resources Management, vol. 18, no. 3, pp. 177199, 2003. 

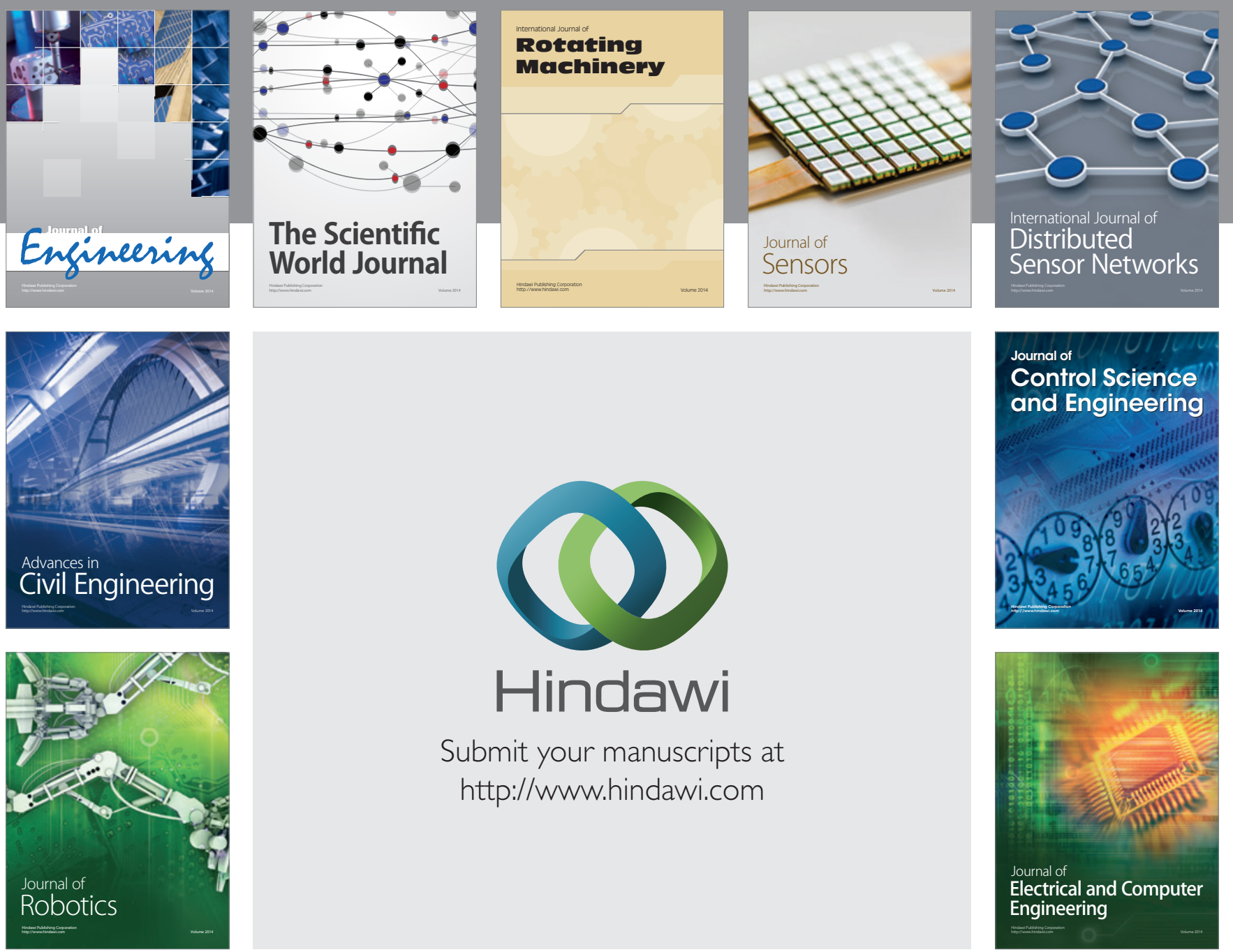

Submit your manuscripts at

http://www.hindawi.com
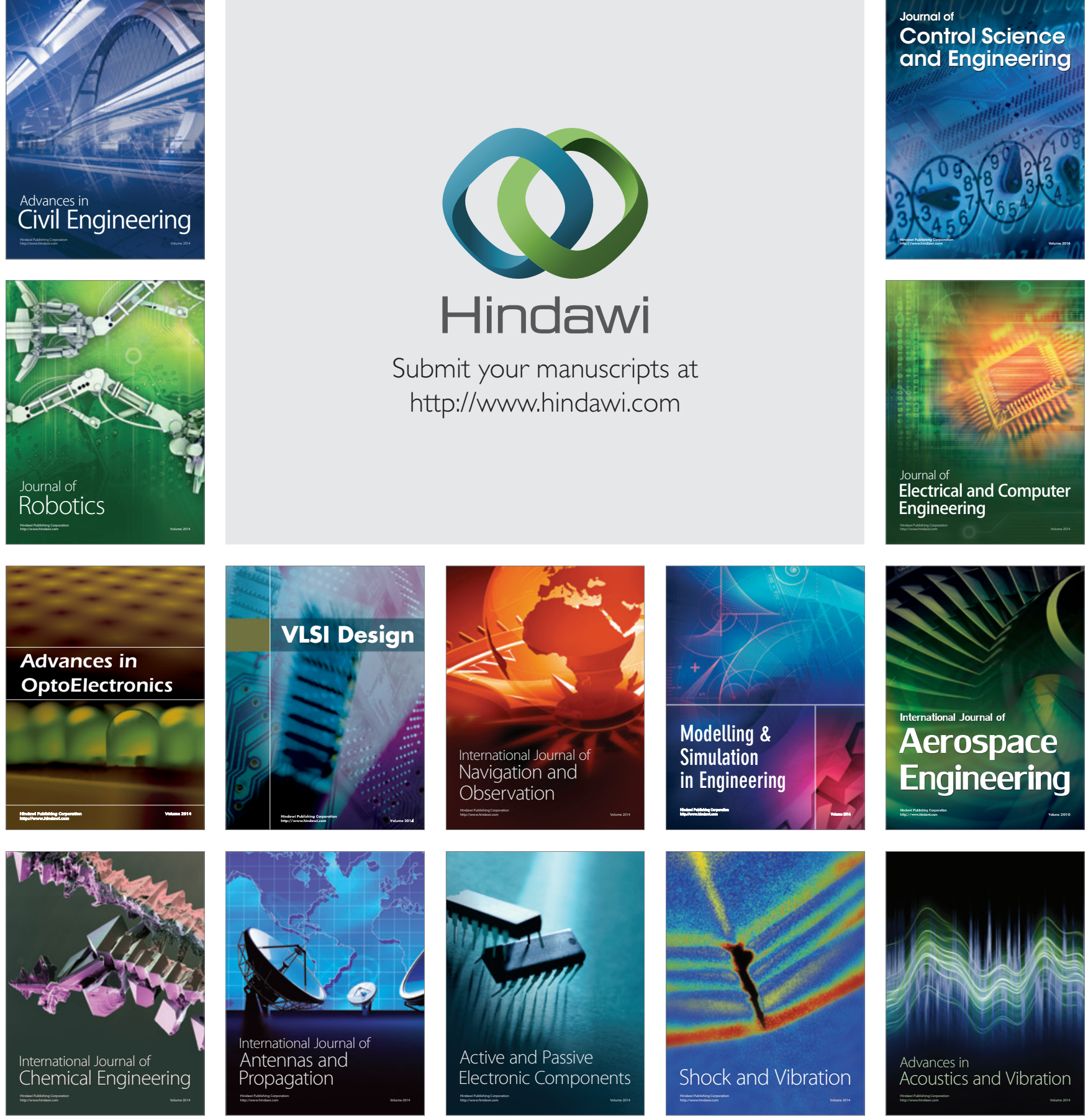